\title{
基于马来酰亚胺的不对称硅氢化一步实现碳手性、轴手性的构建
}

\author{
贺乔星 ${ }^{a, b}$ 黄 正 ${ }^{*, a, b, c}$ \\ ( ${ }^{a}$ 上海科技大学 物质科学与技术学院 上海 201210) \\ $\left({ }^{b}\right.$ 中国科学院上海有机化学研究所 金属有机化学国家重点实验室 上海 200032) \\ ( ${ }^{c}$ 中国科学院杭州高等研究院 化学与材料科学学院 杭州 310024)
}

\section{Single-Step Construction of Carbon Chirality and Axial Chirality by Enantioselective Hydrosilylation of Maleimides}

\author{
He, Qiaoxing ${ }^{a, b} \quad$ Huang, Zheng*, ${ }^{* a, b, c}$ \\ $\left({ }^{a}\right.$ School of Physical Science and Technology, ShanghaiTech University, Shanghai 201210) \\ ( ${ }^{b}$ State Key Laboratory of Organometallic Chemistry, Shanghai Institute of Organic Chemistry, \\ Chinese Academy of Sciences, Shanghai 200032) \\ ( ${ }^{c}$ School of Chemistry and Material Sciences, Hangzhou Institute for Advanced Study, Hangzhou 310024)
}

手性硅烷及其衍生物因其在不对称催化以及功能 材料领域有着非常广泛的应用前景, 近年来越来越受到 合成化学家的重视和广泛关注, 此外手性硅化合物在含 硅候选药物以及手性硅基材料上也被广泛报道 ${ }^{[1-3]}$. 但 是与传统的通过碳硅键的偶联来构筑手性硅化合物相 比，高对映选择性地合成带有极性官能团的手性硅化合 物目前仍然面临巨大的挑战. 例如, 对于活化的烯烃如 $\alpha, \beta$-不饱和羰基化合物，在发生不对称硅氢化反应时, 其主要难点在于反应中不可避免地生成双键还原产物 以及 $\alpha$-加成物和 $\beta$ 加成物等, 除此以外反应还需要考虑 到官能团兼容性以及手性控制等问题 ${ }^{[4-6]}$ (Scheme 1). 因 此如何在更为广泛的底物类型上实现高对映选择性的 硅氢化, 仍然是该研究领域里亟需解决的问题.

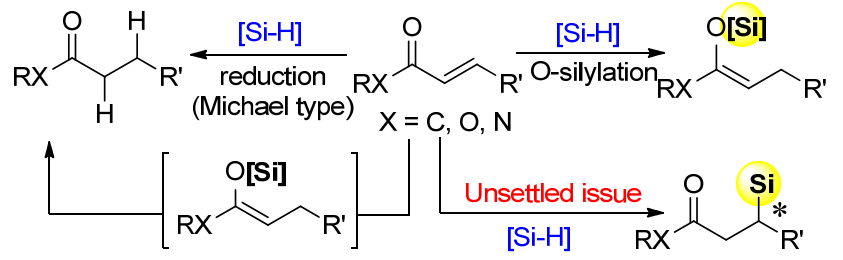

图式 1 传统硅氢化对 EWG-活化烯烃的还原和氧-硅化

Scheme 1 Reduction and $O$-silylation of EWG-activated alkenes for traditional hydrosilylation

近年来，催化不饱和碳碳键的不对称硅氢化为构建 手性硅化合物提供了一种全新的策略 ${ }^{[7-8]}$. 然而目前该
类合成方法局限于末端烯烃或炔烃类底物，对于内烯烃 或活化的烯烃仍然存在比较大的挑战. 马来酰亚胺及其 衍生物是合成化学中一类非常重要的合成砌块，同时存 在于潜在的生物活性分子以及候选药物中 ${ }^{[9-10]}$. 如果能 实现对马来酰亚胺碳碳双键的不对称硅氢化, 将为后期 的具有生物活性的含硅候选药物的笕选提供了可能. 材 料与化学化工学院徐利文课题组 ${ }^{[11]}$ 认为可以通过调节 手性配体的结构及其空腔来调控金属中心的性质，从而 改变不对称硅氢化反应的化学选择性，抑制双键还原等 副反应的发生，使其专一地发生硅碳键偶联反应。

近日该研究团队 ${ }^{[11]}$ 利用 TADDOL-衍生的膦配体活 化的钯催化体系实现了对于马来酰亚胺立体专一性的 硅碳键偶联反应，构筑了一系列手性硅化合物. 值得注 意的是，该方法能够在实现不对称硅氢化的同时，通过 在马来酰亚胺的 $N$-芳基的邻位上引入两个不同位阻大 小取代基以稳定阻转异构体，从而一步构建具有碳手性 和轴手性的有机硅化合物(Scheme 2).

在初始的条件篮选过程中，作者考察了一系列 $(R)$-BINAP, $(R)$-SEGPHOS 和其他膦配体, 发现得到的 均是双键还原产物. 在进一步的配体篮选中作者发现, 采用带有大位阻芳基取代基的 TADDOL-衍生的膦配体 能够以 $93 \%$ 的收率和 $96 \%$ 的 $e e$ 值得到不对称硅氢化产 物. 作者认为手性膦配体空间位阻的排斥作用对于抑制

* Corresponding author. E-mail: huangzh@sioc.ac.cn. Published online September 2, 2020 


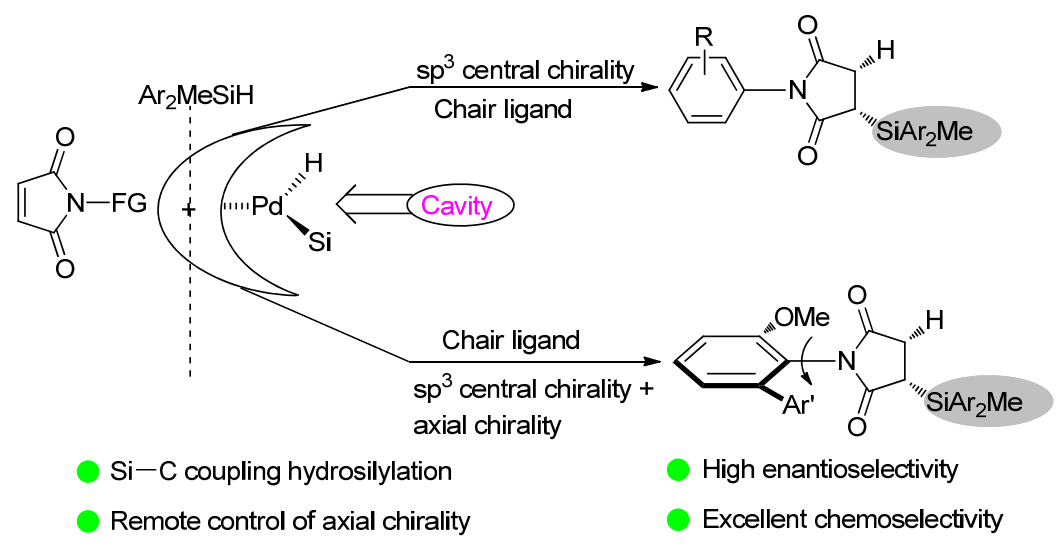

图式 $2 N$-芳基马来酰亚胺催化不对称硅氢化用于远程控制轴手性

Scheme 2 Desymmetric hydrosilylation catalyzed by $N$-aryl-maleimides for remote control of axial chirality

碳碳双键的还原起到了关键作用. 在随后的条件优化 中，作者进一步考察了钯催化剂前体、反应温度及反应 溶剂等因素对反应的影响. 在确定好最优的反应条件 后，作者能够在较为温和的反应条件下以良好的产率高 立体选择性地得到一系列结构多样的手性硅化合物, ee 值最高可达 $99 \%$.

在进一步的底物扩展中, 作者设想, 能否通过在马 来酰亚胺上的芳基邻位引入两个不同位阻大小的取代 基，使得反应在发生不对称硅氢化的同时通过限制 $\mathrm{C}-$ $\mathrm{N}$ 键的旋转达到远程控制轴手性的目的, 最终使得反应 可以一步构筑同时具备碳手性、轴手性的有机硅化合物. 通过对 $N$-芳基上两个取代基大小的调节，作者成功实 现了这一设想, 制备了一系列同时具备碳手性、轴手性
的含硅化合物. 随后作者也进行了克级规模的放大实验 并对其合成的手性硅化合物的应用进行了尝试. 实验结 果证明该类手性硅化合物可以进行一系列丰富的化学 转化(Scheme 3).

综上所述，徐利文课题组创新性地发展了一类新型 的不对称钯催化硅氢加成反应，通过对手性配体结构的 精密调节及优化构建了新型钯催化体系, 成功实现了第 一例对马来酰亚胺立体专一的硅碳键偶联反应，一步构 筑兼具碳手性和轴手性的新型有机硅化合物. 该策略具 有专一的化学选择性和优异的立体选择性、温和的反应 条件和良好的底物普适性等优点. 基于该方法生成的手 性硅化合物能够适用于多样性的转化反应进行丰富的 后续转化.

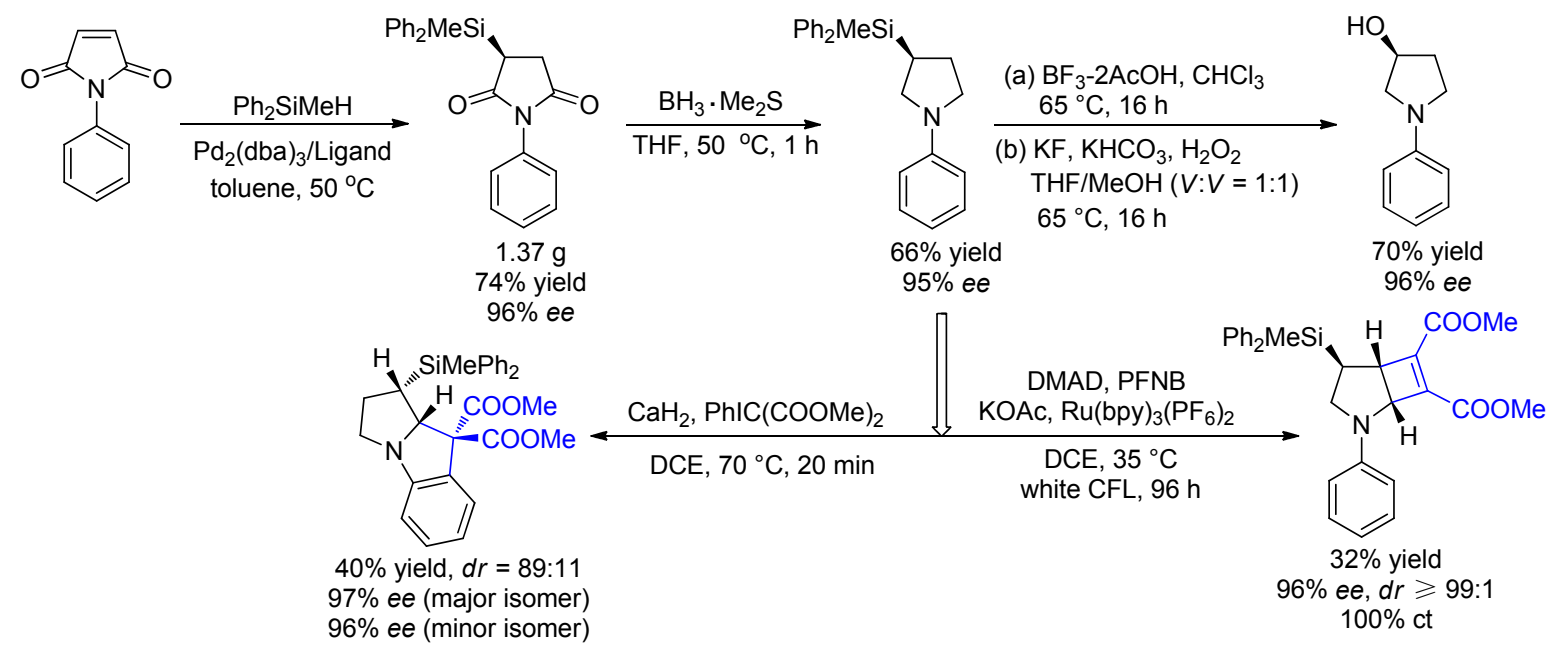

图式 3 含手性硅基琥珀酰亚胺的化学转化

Scheme 3 Transformations of enantiomerically enriched silyl succinimides

\section{References}

[1] Franz, A. K.; Wilson, S. O. J. Med. Chem. 2013, 56, 388.

[2] Singh, S.; Sieburth, S. M. Org. Lett. 2012, 14, 4422.

[3] Ramesh, R.; Reddy, D. S. J. Med. Chem. 2018, 61, 3779.

[4] Sumida, Y.; Yorimitsu, H.; Oshima, K. J. Org. Chem. 2009, 74, 7986.
[5] Benohoud, M.; Tuokko, S.; Pihko, P. M. Chem.-Eur. J. 2011, 17, 8404.

[6] Gandhamsetty, N.; Park, J.; Jeong, J.; Park, S. W.; Park, S.; Chang, S. Angew. Chem., Int. Ed. 2015, 54, 6832.

[7] Wen, H.; Wan, X.; Huang, Z. Angew. Chem., Int. Ed. 2018, 57, 6319. 
[8] Tang, R. H.; Xu, Z.; Nie, Y. X.; Xiao, X. Q.; Yang, K. F.; Xie, J. L.; Guo, B.; Yin, G. W.; Yang, X. M.; Xu, L. W. iScience 2020, 23, 101268.

[9] Li, F.; Li, X.; Wang, Y.; Zhang, X. Angew. Chem., Int. Ed. 2019, 58, 17994.
[10] St Amant, A. H.; Huang, F.; Lin, J.; Rickert, K.; Oganesyan, V.; Lemen, D.; Mao, S.; Harper, J.; Marelli, M.; Wu, H.; Gao, C.; Read de Alaniz, J.; Christie, R. J. Angew. Chem., Int. Ed. 2019, 58, 8489.

[11] Gu, X. W.; Sun, Y. L.; Xie, J. L.; Wang, X. B.; Xu, Z.; Yin, G. W.; Li, L.; Yang, K. F.; Xu, L. W. Nat. Commun. 2020, 11, 2904.

(Zhao, C.) 